International Journal of Pure and Applied Mathematics

Volume 116 No. 1 2017, 153-168

ISSN: 1311-8080 (printed version); ISSN: 1314-3395 (on-line version)

url: http://www.ijpam.eu

doi: 10.12732/ijpam.v116i1.16

\title{
MORE ON WEAK DECOMPOSITION OF CONTINUITY
}

\author{
Ennis Rosas ${ }^{1}$, Carlos Carpintero ${ }^{2}$, John Moreno $^{3}$, Jose Sanabria ${ }^{4}$ \\ ${ }^{1,2}$ Department of Mathematics \\ Universidad de Oriente \\ Núcleo De Sucre-Cumaná, VENEZUELA \\ ${ }^{1}$ Department of Natural Sciences and Exact \\ Universidad de la Costa \\ Barranquilla, COLOMBIA \\ ${ }^{3}$ Departamento de Ciencias Naturales y Exacta \\ Universidad de la Costa \\ Barranquilla, COLOMBIA \\ ${ }^{4}$ Department of Mathematics \\ Universidad de Oriente \\ Núcleo De Sucre Cumaná \\ Venezuela and Program of Mathematics \\ Universidad del Atlántico \\ Barranquilla, COLOMBIA
}

\begin{abstract}
Using the notion of $w$-space on a set $X$ and the concept of locally $w$-semi open set, we introduce, study and characterize the notions of $w$-s-Kernel of a subset $A$ of $X$. Also we introduce and study a new forms of weak decomposition of continuity.
\end{abstract}

AMS Subject Classification: 54C05, 54C08, 54C10

Key Words: $\mu$-st-set, $\mu$-sB-set, $(\mu, \sigma)$-s-continuous functions, $w$-s- $\operatorname{Ker}(A)$

\section{Introduction and Preliminaries}

In the last years, different variants of open sets are being studied. Recently, a

Received: June 13, 2017

Revised: $\quad$ July 23, 2017

Published: August 30, 2017

(c) 2017 Academic Publications, Ltd.

url: www.acadpubl.eu

$\S_{\text {Correspondence author }}$ 
significant contribution to the theory of generalized open sets have been presented by A. Császár [1], [2], [3]. Specifically, in 2002, A. Császár [1], introduced the notions of generalized topology and generalized continuity. It is observed that a large numbers of articles are devoted to the study of generalized open sets and certain type of sets associated to a topological spaces, containing the class of open sets and possessing properties more or less to those open sets. Bishwambhar. et al. [4] studied some type of decomposition of continuity using generalized topologies and in [5], studied some weak forms of continuity. Rosas E. et al. in [10], give a new theory of decomposition of continuous functions using generalized topologies. In 2015, W. K. Min et al. [7], introduced and studied the notions of weak structures on a nonempty set $X$. In 2016, W. K. Min et al. introduced the notions of $w$-semiopen sets and $w$-semi continuity in $w$-spaces. Later in 2017, W. K. Min in [6], introduced and studied the notions of weakly $w_{\tau} g$-closed set and weakly $w_{\tau} g$-open set as a generalization of the $w_{\tau} g$-closed set and $w_{\tau} g$-open set in associated w-spaces. E. Rosas et al in [9], introduce the concepts of locally $w$-regular closed sets and locally $w$-semi regular semi closed and a new weak decomposition of some type of weak continuity functions are studied and characterized. In this article, using the notion of $w$-semi open set, we introduce the concept of locally $w$-semi open set as a generalization of locally $w$-closed and give a new theory of weak decomposition of continuity and some weak form of continuity are studied. Throughout this paper $\operatorname{cl}(A)$ (respectively $\operatorname{int}(A)$ ) denotes the closure (respectively interior) of $A$ in a topological space $X$.

\section{Preliminaries}

Definition 2.1. [7] Let $X$ be a nonempty set. A subfamily $w_{X}$ of the power set $P(X)$ is called a weak structure on $X$ if it satisfies the following :

1. $\emptyset \in w_{X}$ and $X \in w_{X}$.

2. For $U_{1}, U_{2} \in w_{X}, U_{1} \cap U_{2} \in w_{X}$

Then the pair $\left(X, w_{X}\right)$ is called a $w$-space on $X$. An element $U \in w_{X}$ is called $w$-open set and the complement of a $w$-open set is a $w$-closed set

Definition 2.2. [7] Let $\left(X, w_{X}\right)$ be a $w$-space. For a subset $A$ of $X$,

1. The $w$-closure of $A$ is defined as $w C(A)=\bigcap\left\{F: A \subseteq F, X \backslash F \in w_{X}\right\}$.

2. The $w$-interior of $A$ is defined as $w I(A)=\bigcup\left\{U: U \subseteq A, U \in w_{X}\right\}$. 
Theorem 2.3. [7] Let $\left(X, w_{X}\right)$ be a $w$-space on $X$. A, B subsets of $X$. Then the following hold:

1. If $A \subseteq B$, then $w I(A) \subseteq w I(B)$ and $w C(A) \subseteq w C(B)$.

2. $w I(w I(A))=w I(A)$ and $w C(w C(A))=w C(A)$

3. $w C(X \backslash A)=X \backslash w I(A)$ and $w I(X \backslash A)=X \backslash w C(A)$

4. If $A$ is $w$-closed (resp.w-open), then $w C(A)=A(\operatorname{resp} . w I(A)=A)$

Definition 2.4. [8] Let $\left(X, w_{X}\right)$ be a $\omega$-space on $X$. A subset $A$ of $X$ is called $w$-semi open if $A \subseteq w C(w I(A))$. The complement of a w-semi open set is called w-semi closed.

The collection of all $w$-semi open sets is denoted by $w S O\left(X, w_{X}\right)$ and the collection of all $w$-semi closed sets is denoted by $w S C\left(X, w_{X}\right)$

Definition 2.5. [8] Let $\left(X, w_{X}\right)$ be a $w$-space on $X$. For a subset $A$ of $X$

1. The $w$-semi closure of $A$ is defined as $w s C(A)=\bigcap\{F: A \subseteq F, X \backslash F \in$ $\left.w S O\left(X, w_{X}\right)\right\}$.

2. The $w$-semi interior of $A$ is defined as $w s I(A)=\bigcup\{U: U \subseteq A, U \in$ $\left.w S O\left(X, w_{X}\right)\right\}$.

Theorem 2.6. [8] Let $\left(X, w_{X}\right)$ be a $w$-space on $X$. A, B subsets of $X$. Then the following hold:

1. $w s I(A) \subseteq A$ and $A \subseteq w s C(A)$.

2. If $A \subseteq B$, then $w s I(A) \subseteq w s I(B)$ and $w s C(A) \subseteq w s C(B)$.

2. $w s I(w s I(A))=w s I(A)$ and $w s C(w s C(A))=w s C(A)$.

3. $w s C(X \backslash A)=X \backslash w s I(A)$ and $w s I(X \backslash A)=X \backslash w s C(A)$.

4. $A$ is $w$-semi closed (resp. $w$-semi open), if and only if $w s C(A)=A$ (resp. $w s I(A)=A)$.

Theorem 2.7. [8] Let $\left(X, w_{X}\right)$ be a $w$-space on $X$ and $A$ a subset of $X$. Then wsC $(A)$ is an $w$-semi closed.

Theorem 2.8. Let $\left(X, w_{X}\right)$ be a $w$-space on $X$ and $A$ a subset of $X$. Then $A \cup w I(w C(A)) \subseteq w s C(A)$. 
The following example shows that the reverse contention in the above theorem is not necessarily true.

Example 2.9. Let $X=N$ be the set of natural numbers. Define $w_{X}=$ $\{\emptyset,\{1\}, N\} \cup P(\{2 n: n \in N\})$. The set of $w$-closed sets $=\{\emptyset, N, N \backslash\{1\}\} \cup\left\{A^{c}\right.$ : $A \in P(\{2 n: n \in N\})\}$. The set of $w$-semiopen sets $=\left\{\emptyset,\{1\}, N, F_{1}, F_{2}\right\} \cup$ $P(\{2 n: n \in N\})$ where $F_{1} \cap\{2 n: n \in N\} \neq \emptyset$ and $1 \in F_{2}$. If we take $A=\{3\}$, $w s C(A)=\{3\}, w C(A)=\{2 n+1: n \in N\}$ and $w I(w C(A))=\{1\}$. Observe that $A \cup w I(w C(A))=\{1,3\} \supset\{3\}=w s C(A)$.

Theorem 2.10. Let $\left(X, w_{X}\right)$ be a $w$-space on $X$ and $A, B$ subsets of $X$. Then

1. $x \in w s C(A)$ if and only if $A \cap V \neq \emptyset$, for every $w$-semiopen set $V$ containing $x$.

2. $w s C(A \cap B)=w s C(A) \cap w s C(B)$.

\section{New Types of $w$-Closed Sets}

Throughout this paper $\left(X, w_{X}, \tau\right)$ a weak topological space denotes $\left(X, w_{X}\right)$ is a $w$-space and $(X, \tau)$ is a topological space.

Definition 3.1. [4] Let $\left(X, w_{X}, \tau\right)$ be a weak topological space. A subset $A$ of $X$ is called locally $w$-closed if $A=U \cap F$ where $U \in \tau$ and $F$ is $w$-closed.

Remark 3.2. If $\left(X, w_{X}, \tau\right)$ is a weak topological space, then every open set as well as a $w$-closed set is locally $w$-closed.

Theorem 3.3. Let $\left(X, w_{X}, \tau\right)$ be a weak topological space. If $A \subseteq X$ is locally $w$-closed then there exists an open set $U$ such that $A=U \cap w C(A)$.

Proof. Let $A$ be a locally $w$-closed subset of $X$, then $A=U \cap F$, where $U \in \tau$ and $F$ is $w$-closed. It follows that $A=A \cap U \subseteq U \cap w C(A) \subseteq U \cap w C(F)=$ $U \cap F=A$. In consequence, $A=U \cap w C(A)$.

Example 3.4. In Example 3.12, $\{a, b\}=\{a, b, c\} \cap w s C(\{a, b\})$, but $\{a, b\}$ is not a locally $w$-closed set.

Definition 3.5. Let $\left(X, w_{X}, \tau\right)$ be a weak topological space. A subset $A$ of $X$ is called: 
1. $w$-t-set if $\operatorname{int}(A)=\operatorname{int}(w C(A))$.

2. $w$-B-set if $A=U \cap V, U \in \tau, V$ is a $w$-t-set.

3. $w^{*}$-open set if $A \subseteq \operatorname{cl}(w I(A))$.

4. $w$-open set if $A \subseteq \operatorname{int}(w C(A))$.

Definition 3.6. Let $\left(X, w_{X}, \tau\right)$ be a weak topological space. A subset $A$ of $X$ is called generalized $w$-closed or simple a gw-closed if $w C(A) \subseteq U$ whenever $A \subseteq U$ and $U \in \tau$.

Definition 3.7. $[9]$ Let $\left(X, w_{X}, \tau\right)$ be a weak topological space. A subset $A$ of $X$ is called locally $w$-semi closed or simple locally $w$-s-closed if $A=U \cap F$ where $U \in \tau$ and $F$ is $w$-semi closed.

Remark 3.8. If $\left(X, w_{X}, \tau\right)$ is a weak topological space, then every open set as well as a $w$-semi closed set is locally $w$-semi closed, also every locally $w$-closed set is locally $w$-semi closed.

Definition 3.9. Let $\left(X, w_{X}, \tau\right)$ be a weak topological space. A subset $A$ of $X$ is called:

1. $w$-st-set if $\operatorname{int}(A)=\operatorname{int}(w s C(A))$.

2. $w$-sB-set if $A=U \cap V, U \in \tau, V$ is a $w$-st-set.

3. $w^{*}$-semi open set (briefly $w^{*}$-s-open set) if $A \subseteq \operatorname{cl}(w s I(A))$.

4. $w$ '-semi open set (briefly $w^{\prime-S-o p e n ~ s e t) ~ i f ~} A \subseteq \operatorname{int}(w s C(A))$.

The complement of a $w^{*}$-semi open set is called $w^{*}$-semi closed set (briefly $w^{*}$-s-closed set).

The following theorems characterizes the locally $w$-semi closed sets.

Theorem 3.10. Let $\left(X, w_{X}, \tau\right)$ be a weak topological space. $A \subseteq X$ is locally $w$-semi closed if and only if $X-A$ is the union of a closed set and a $w$-semi open set.

Proof. Suppose that $A$ is locally $w$-semi closed, then $A=U \cap F$ where $U \in \tau$ and $F$ is $w$-semi closed. It follows that $X-A=X \cap(U \cap F)^{c}=\left(U^{c} \cup F^{c}\right)=$ $(X-U) \cup(X-F)$. Conversely, suppose that $X-A=W \cup G, W$ closed set and $G w$-semi open. Then $A=X-(X-A)=X-(W \cup G)=(X-W) \cap(X-G)$. 
Theorem 3.11. Let $\left(X, w_{X}, \tau\right)$ be a weak topological space. $A \subseteq X$ is locally $w$-semi closed if and only if there exists an open set $U$ such that $A=U \cap w s C(A)$.

Proof. Let $A$ be a locally $w$-semi closed subset of $X$, then $A=U \cap F$, where $U \in \tau$ and $F$ is $w$-semi closed. It follows that $A=A \cap U \subseteq U \cap w s C(A) \subseteq$ $U \cap w s C(F)=U \cap F=A$. In consequence, $A=U \cap w s C(A)$. Conversely, since $w s C(A)$ is a $w$-semi closed, it follows that $A$ is locally $w$-semi closed.

In the following example, we can see that there exists a locally $w$-semi closed set that is not open as well as $w$-semi closed, $w$-st-set that is not $w$-s-set, $w$-sB-set that is not $w$-B-set.

Example 3.12. Let $X=\{a, b, c, d\}, \tau=\{\emptyset, X,\{a, b, c\},\{c, d\},\{c\}\}$ and $w_{X}=\{\emptyset, X,\{a, b\},\{b, c\},\{b\}\}$ be a weak structure on $(X, \tau)$. Then:

1. $w$-closed sets $=\{\emptyset, X,\{c, d\},\{a, d\},\{a, c, d\}\}$.

2. locally $w$-closed $=\{\emptyset, X,\{a, b, c\},\{a, c, d\},\{c, d\},\{c\},\{a, d\},\{a\}$, $\{a, c\},\{d\}\}$.

3. $w S O\left(X, w_{X}\right)=\{\emptyset, X,\{b\},\{a, b\},\{b, c\},\{b, d\},\{a, b, c\},\{a, b, d\}$, $\{a, c, d\}\}$.

4. $w S C\left(X, w_{X}\right)=\{\emptyset, X,\{a, c, d\},\{c, d\},\{a, d\},\{a, c\},\{d\},\{c\},\{b\}\}$.

5. locally $w$-s-closed $=\{\emptyset, X,\{a, b, c\},\{c, d\},\{c\},\{a, c, d\},\{c, d\},\{a, d\}$, $\{a, c\},\{d\},\{b\},\{a\}\}$.

6. $w$-t-set $=\{\emptyset, X,\{a\},\{d\},\{a, d\},\{c, d\},\{a, c, d\}\}$.

7. $w$-st-set $=\{\emptyset, X,\{a\},\{b\},\{d\},\{a, d\},\{a, c\},\{c, d\},\{a, c, d\}\}$.

8. $w$-B-set $=\{\emptyset, X,\{a, b, c\},\{c, d\},\{c\},\{a\},\{d\},\{a, d\},\{c, d\},\{a, c, d\}$, $\{a, c\}\}$.

9. $w$-sB-set $=\{\emptyset, X,\{a, b, c\},\{c, d\},\{c\},\{a\},\{b\},\{d\},\{a, d\},\{a, c\}$, $\{a, c, d\}\}$.

10. $w^{*}$-open $=\{\emptyset, X,\{b\},\{a, b\},\{b, c\},\{a, b, c\},\{b, c, d\}\}$.

11. $w^{*}$-s-open $=\{\emptyset, X,\{b\},\{a, b\},\{b, c\},\{a, b, c\},\{a, b, d\},\{a, c, d\}$, $\{b, c, d\}\}$. 
12. $w$-open $=\{\emptyset, X,\{b\},\{c\},\{a, b\},\{b, c\}\{b, d\},\{c, d\},\{a, b, c\}$, $\{b, c, d\}\}$.

13. w'-s-open $=\{\emptyset, X,\{a, b\},\{b, c\},\{b, d\},\{c, d\}\{a, b, d\},\{a, b, d\}$, $\{a, c, d\},\{b, c, d\}\}$.

The following examples will be useful to better understand the development of this article.

Example 3.13. Let $X=\{a, b, c\}, \tau=\{\emptyset, X,\{a\},\{b\},\{a, b\}\}$ and $w_{X}=$ $\{\emptyset, X,\{a\},\{a, c\}\}$ be a weak structure on $(X, \tau)$. Then:

1. $w$-closed sets $=\{X,\{b, c\},\{b\}\}$.

2. locally $w$-closed $=\{\emptyset, X,\{a\},\{b\},\{a, b\},\{b, c\}\}$.

3. $w S O(X, w(X))=\{\emptyset, X,\{a\},\{b\},\{a, b\},\{a, c\}\}$.

4. $w S C(X, w(X))=\{\emptyset, X,\{b, c\},\{c\},\{b\},\{a, c\}\}$.

5. locally $w$-s-closed $=\{\emptyset, X,\{b, c\},\{c\},\{b\},\{a\},\{a, b\},\{a, c\}\}$.

6. $w$-t-set $=\{X,\{b\},\{b, c\}\}$.

7. $w$-st-set $=\{\emptyset, X,\{b\},\{c\},\{a\},\{b, c\},\{a, c\}\}$.

8. $w$-B-set $=\{\emptyset, X,\{b\},\{a\},\{b, c\},\{a, b\}\}$.

9. $w$-sB-set $=\{\emptyset, X,\{b\},\{c\},\{a\},\{a, b\},\{a, c\},\{b, c\}\}$.

10. $w^{*}$-open $=\{\emptyset,\{a\},\{b\},\{a, c\}\}$.

11. $w^{*}$-s-open $=\{\emptyset, X,\{a\},\{b\},\{a, b\},\{a, c\},\{b, c\}\}$.

12. $w$-open $=\{\emptyset, X,\{a\},\{b\},\{a, b\},\{a, c\}\}$.

13. $w^{\prime}$-S-open $=\{\emptyset, X,\{a\},\{b\},\{a, b\}\}$.

In a weak topological space $\left(X, w_{X}, \tau\right)$, always $w s C(A) \subseteq w C(A)$ and $w I(A) \subseteq w s I(A)$ for all $A \subseteq X$. Therefore, we obtain the relationship between the sets given in Definitions 3.5 and 3.7.

Theorem 3.14. Let $\left(X, w_{X}, \tau\right)$ be a weak topological space and $A \subset X$. Then the following holds:

1. If $A$ is a $w$-t-set, then $A$ is a $w$-st-set. 
2. If $A$ is a $w$-B-set, then $A$ is a $w$-sB-set.

3. If $A$ is a $w^{*}$-open, then $A$ is a $w^{*}$-s-open.

4. If $A$ is a $w^{\prime}-$-sopen, then $A$ is a $w^{\prime}$-open.

Example 3.15. Let $X=\{a, b, c\}$ with $\tau=\{\emptyset, X,\{a\},\{b\},\{a, b\}\}$ and weak structure $w_{X}=\{\emptyset, X,\{b, c\}\}$ and on $(X, \tau)$. Observe that $\tau$ is not contained in $w$. If we take $A=\{a, b\}, A$ is locally $w$-semi closed, because $A=\{a, b\} \cap X, w s C(A)=X, w s C(A)-A=\{c\}$ is not $w$-semi closed. $A \cup(X-w s C(A))=A=\{a, b\}$ is not a $w$-semi open set, also $A$ is not contained in $w s I(A \cup(X-w s C(A)))$, because $A \cup(X-w s C(A))=\{a, b\}$ and $w s I(\{a, b\})=\emptyset$.

In the case that $\tau \subset w_{X}$, we have the following theorem.

Theorem 3.16. Let $\left(X, w_{X}, \tau\right)$ be a weak topological space and $\tau \subset w_{X}$. If $A$ is locally $w$-semi closed, then:

1. $w s C(A)-A$ is $w$-semi closed.

2. $A \cup(X-w s C(A))$ is $w$-semi open set.

3. $A$ is contained in $w s I(A \cup(X-w s C(A)))$.

Proof. 1.- Suppose that $A$ is a locally $w$-semi closed subset of $X$, then there exists an open set $U$ such that $A=U \cap w s C(A)$. It follows that: $w s C(A)-A=$ $w s C(A)-(U \cap w s C(A))=w s C(A) \cap(X-(U \cap w s C(A)))=w s C(A) \cap((X-$ $U) \cup(X-w s C(A)))=w s C(A) \cap(X-U) \cup w s C(A) \cap(X-w s C(A))=$ $w s C(A) \cap(X-U)$. Now, as $w s C(A)$ is $w$-semi closed, $X-U$ is closed and $\tau \subset \mu$, we obtain that $X-U$ is $w$-semi closed and then $\operatorname{ws} C(A) \cap(X-U)$ is $w$-semi closed.

2.- Using (1), ws $C(A)-A$ is $w$-semi closed, then its complement $X-(w s C(A)-$ $A)$ is $w$-semi open, but $X-(w s C(A)-A)=X-(w s C(A) \cap(X-A)=$ $A \cup(X-w s C(A))$.

3.- Using (2), $A \subset(A \cup(X-w s C(A)))=w s I(A \cup(X-w s C(A)))$.

Definition 3.17. Let $\left(X, w_{X}, \tau\right)$ be a weak topological space. A subset $A$ of $X$ is called generalized $w$-closed or simple a gw-closed if $w C(A) \subseteq U$ whenever $A \subseteq U$ and $U \in \tau$.

Definition 3.18. Let $\left(X, w_{X}, \tau\right)$ be a weak topological space. A subset $A$ of $X$ is called generalized $w$-semi closed or simple a gw-s-closed if $w s C(A) \subseteq U$ whenever $A \subseteq U$ and $U \in \tau$. 
Remark 3.19. In a weak topological space $\left(X, w_{X}, \tau\right)$, every generalized $w$-closed set is a generalized $w$-semi closed set, but the converse is not necessarily true as we can see in the following example.

Example 3.20. In Example 3.12,

1. locally $w$-closed $=\{\emptyset, X,\{a, b, c\},\{a, c, d\},\{c, d\},\{c\},\{a, d\}$, $\{a\},\{a, c\},\{d\}\}$

2. gw-closed $=\{\emptyset, X,\{d\},\{a, d\},\{b, d\},\{c, d\},\{a, c, d\},\{a, b, d\}$, $\{b, c, d\}\}$.

3. $w$-closed sets $=\{\emptyset, X,\{c, d\},\{a, d\},\{a, c, d\}\}$.

4. locally $w$-s-closed $=\{\emptyset, X,\{a, b, c\},\{c, d\},\{c\},\{a, c, d\},\{a, d\}$, $\{a, c\},\{d\},\{b\},\{a\}\}$.

5. gw-s-closed $=\{\emptyset, X,\{a\},\{b\},\{c\},\{d\},\{a, d\},\{a, c\},\{c, d\}$, $\{a, b, d\},\{a, c, d\},\{b, c, d\}\}$.

6. $w S C\left(X, w_{X}\right)=\{\emptyset, X,\{a, c, d\},\{c, d\},\{a, d\},\{a, c\},\{d\},\{c\}$, $\{b\}\}$.

The following theorems characterize: the $w$-closed sets in terms of gw-closed sets and locally $w$-closed sets and the $w$-semi closed sets in terms of gw-semi closed sets and locally $w$-semi closed sets.

Theorem 3.21. Let $\left(X, w_{X}, \tau\right)$ be a weak topological space. $A \subset X$ is $w$-closed if and only if $A$ is gw-closed and locally $w$-closed.

Proof. Suppose that $A$ is $w$-closed in $X$ and $A \subset U$, with $U \in \tau$. Since $A=w C(A)$, we obtain that $A$ is gw-closed and locally $w$-closed.

Conversely, suppose that $A$ is gw-closed and locally $w$-closed, then $A=U \cap F$, where $U \in \tau$ and $F$ is $w$-closed, therefore, $A \subset U$ and $A \subset F$, in consequence, $w C(A) \subset U$ and $w C(A) \subset F$ and hence $w C(A) \subset U \cap F=A$. So $A$ is $w$-closed.

Theorem 3.22. Let $\left(X, w_{X}, \tau\right)$ be a weak topological space. $A \subset X$ is $w$-semi closed if and only if $A$ is gw-semi closed and locally $w$-semi closed.

Proof. Suppose that $A$ is $w$-semi closed in $X$ and $A \subset U$, with $U \in \tau$. Since $A=w s C(A)$, we obtain that $A$ is gw-semi closed and locally $w$-semi closed. Conversely, suppose that $A$ is gw-semi closed and locally $w$-semi closed, then $A=U \cap F$, where $U \in \tau$ and $F$ is $w$-semi closed, therefore, $A \subset U$ and $A \subset F$, in 
consequence, $w s C(A) \subset U$ and $w s C(A) \subset F$ and hence $w s C(A) \subset U \cap F=A$. So $A$ is $w$-semi closed.

Theorem 3.23. Let $\left(X, w_{X}, \tau\right)$ be a weak topological space and $A, B$ subsets of $X$.

1. $A$ is a $w$-st-set if and only if $A$ is a $w^{*}$-s-closed.

2. If $A$ is $w$-semi closed, then $A$ is $w$-st-set.

3. If $A$ and $B$ are $w$-st-sets, then $A \cap B$ is $w$-st-set.

4. If $A$ is $w$-st-set, then $A$ is $w$-sB-set.

5. Every locally $w$-semi closed set is $w$-sB-set.

Proof. 1-. Suppose that $A$ is a $w$-st-set, then $\operatorname{int}(A)=\operatorname{int}(w s C(A))$ and hence $\operatorname{int}(w s C(A)) \subset A$, in consequence, $A$ is a $w^{*}$-s-closed. Conversely, if $A$ is a $w^{*}$-s-closed, then $\operatorname{int}(w s C(A)) \subset A$ and hence

$\operatorname{int}(w s C(A)) \subset \operatorname{int}(A) \subset A \subset \operatorname{int}(w s C(A))$. Therefore, int $(w s C(A)) \subset$ $\operatorname{int}(A) \subset \operatorname{int}(A) \subset \operatorname{int}(w s C(A))$. In consequence, $\operatorname{int}(w s C(A))=A$, so $A$ is a $w$-st-set.

2-. If $A$ is $w$-semi closed, then $A=w s C(A)$, and hence $\operatorname{int}(A)=\operatorname{int}(w s C(A))$. Therefore, $A$ is $w$-st-set.

3-. Suppose that $A$ and $B$ are $w$-st-sets. Since $A \cap B \subseteq w s C(A \cap B)$, we obtain that $\operatorname{int}(A \cap B) \subseteq \operatorname{int}(w s C(A \cap B)) \subseteq \operatorname{int}(w s C(A) \cap$ wsC $(B))=$ $\operatorname{int}(w s C(A)) \cap \operatorname{int}(w s C(B))=\operatorname{int}(A) \cap \operatorname{int}(B)=\operatorname{int}(A \cap B)$. In consequence, $\operatorname{int}(A \cap B)=\operatorname{int}(w s C(A \cap B))$.

4-. Since $X \in \tau$ and $A=A \cap X$, then $A$ is a $\mu$-sB-set.

5-. Suppose that $A$ is locally $w$-semi closed set of $X$, then $A=U \cap F$, where $U \in \tau$ and $F$ is a $w$-semi closed. Using (2), $F$ is $w$-st-set, then by (4), follows that $A=U \cap F$, where $U \in \tau$ and $F$ is a $w$-st-set and therefore, $A$ is $w$-sB-set.

In the following examples show that the converse of the above theorem is not necessarily true.

Example 3.24. In Example 3.12, $\{c\}$ is $w$-sB-set, but is not $w$-st-set. Also $\{a\}$ is $w$-st-set, but is not $w$-semi closed.

Example 3.25. Let $X=\{a, b, c\}, \tau=\{\emptyset, X,\{c\}\}$ and $w_{X}=\{\emptyset, X,\{a\}$, $\{a, b\}\}$. Then $\{\mathrm{a}\}$ is a $w$-sB-set but is not locally $w$-semi closed. 
Theorem 3.26. Let $\left(X, w_{X}, \tau\right)$ be a weak topological space. $A \subset X$ is open if and only if $A$ is $w^{\prime}$-S-open and $w$-sB-set.

Proof. Let $A$ be an open set, then $A=\operatorname{int}(A) \subseteq \operatorname{int}(w s C(A))$ and hence, $A$ is $w$-s-open set. Since $A=A \cap X$, where $X$ is $w$-st-set, then $A$ is $w$-sB-set. Conversely, since $A$ is a $w$-sB-set, $A=U \cap V$, where $U \in \tau$ and $V$ is a $w$-stset. By hypothesis, $A \subseteq \operatorname{int}(w s C(A))=\operatorname{int}(w s C(U \cap V)) \subseteq \operatorname{int}(w s C(U) \cap$ $w s C(V))=\operatorname{int}(w s C(U)) \cap \operatorname{int}(w s C(V))=\operatorname{int}(w s C(U)) \cap \operatorname{int}(V)$. But $A=$ $U \cap V=(U \cap V) \cap U \subseteq(\operatorname{int}(w s C(U)) \cap \operatorname{int}(V)) \cap U=(\operatorname{int}(w s C(U)) \cap U \cap$ $\operatorname{int}(V))=U \cap \operatorname{int}(V) \subseteq U \cap V=A$. Therefore, $A$ is an open set.

Example 3.27. In Example 3.12, $\{b, c, d\}$ is $w$-s-open but not $w$-sB-set. In the same form, $\{a\}$ is $w$-sB-set but is not $w^{\prime}$-s-open, in consequence, is not open.

\section{4. $(w, \sigma)$-s-Continuous Functions}

Definition 4.1. Let $\left(X, w_{X}, \tau\right)$ be a weak topological space and $(Y, \sigma)$ be a topological space. A function $f:(X, \tau) \rightarrow(Y, \sigma)$ is said to be $(w, \sigma)$-continuous if $f^{-1}(V)$ is $w$-open in $X$ for each open set $V$ of $Y$.

Definition 4.2. Let $\left(X, w_{X}, \tau\right)$ be a weak topological space and $(Y, \sigma)$ be a topological space. A function $f:(X, \tau) \rightarrow(Y, \sigma)$ is said to be $(w, \sigma)$-scontinuous if $f^{-1}(V)$ is $w$-semi open in $X$ for each open set $V$ of $Y$.

Theorem 4.3. Every $(w, \sigma)$-continuous function is $(\mu, \sigma)$-s-continuous but not conversely.

Example 4.4. Let $X=\mathbb{R}$ be the set of real numbers, $w_{X}=\{\emptyset, \mathbb{R}, \mathbb{R} \backslash \mathbb{Q}\}$ and $\tau=\sigma=\{\emptyset, \mathbb{R}, \mathbb{Q}\}$ where $\mathbb{Q}$ denotes the set of all rational numbers and $\mathbb{R} \backslash \mathbb{Q}$ denotes the set of all irrational numbers. Define $f:(\mathbb{R}, \tau) \rightarrow(\mathbb{R}, \sigma)$ as the identity function. Then $f$ is $(w, \sigma)$-s-continuous but not $(w, \sigma)$-continuous.

Theorem 4.5. Let $\left(X, w_{X}, \tau\right)$ be a weak topological space, $(Y, \sigma)$ be a topological space and $f:(X, \tau) \rightarrow(Y, \sigma)$ a function. Then the following are equivalent :

1. $f$ is $(w, \sigma)$-s-continuous.

2. For each $x \in X$ and each open set $V$ of $Y$ with $f(x) \in V$, there exists a $w$-semi open set $U$ containing $x$ such that $f(U) \subseteq V$. 
3. For each $x \in X$ and each open set $V$ of $Y$ with $f(x) \in V, f^{-1}(V)$ is a $w$-semi open neighborhood of $x$.

4. The inverse image of each closed set in $Y$ is $w$-semi closed.

5. $w s C\left(f^{-1}(B)\right) \subseteq f^{-1}(c l(B))$ for every $B \subseteq Y$.

6. $f(w s C(A)) \subseteq \operatorname{cl}(f(A))$ for every $A \subseteq X$.

7. $f^{-1}(\operatorname{int}(B)) \subseteq w s I\left(f^{-1}(B)\right)$ for every $B \subseteq Y$.

Proof. 1. $\Rightarrow$ 2. Let $x \in X$ and $V$ any open set in $Y$ such that $f(x) \in V$. Since $f$ is $(w, \sigma)$-s-continuous, $f^{-1}(V)$ is $w$-semi open. By putting $U=f^{-1}(V)$, $x \in U$ and $f(U) \subseteq V$.

2. $\Rightarrow 3$. Let $x \in X$ and $V$ an open in $Y$ such that $f(x) \in V$. By 2, there exists a $w$-semi open set $U$ containing $x$ such that $f(U) \subseteq V$. So each $x \in U \subseteq f^{-1}(V)$ and hence $f^{-1}(V)$ is a $w$-semi open neighborhood of $x$.

3. $\Rightarrow 1$. Let $x \in X$ and $V$ an open in $Y$ such that $f(x) \in V$. By 3, $f^{-1}(V)$ is a $w$-semi open neighborhood of $x$. Thus for each $x \in f^{-1}(V)$, there exists a $w$-semi open set $U_{x}$ containing $x$ such that $x \in U_{x} \subseteq f^{-1}(V)$. Hence $f^{-1}(V)=\cup_{x \in f^{-1}(V)} U_{x}$ and so $f^{-1}(V) \in w S O(X)$.

1. $\Leftrightarrow 4$. It is obvious.

1. $\Rightarrow 5$. Let $B$ be a subset of $Y$. Since $\operatorname{cl}(B)$ is closed and $f$ is $(w, \sigma)$-scontinuous, $f^{-1}(\operatorname{cl}(B)$ is $w$-semi closed. Therefore,

$$
w s C\left(f^{-1}(B)\right) \subseteq w s C\left(f^{-1}(\operatorname{cl}(B))\right)=f^{-1}(\operatorname{cl}(B)) .
$$

5 . $\Rightarrow 6$. Let $A$ be a subset of $X$. By 5 , we have

$$
w s C\left(f^{-1}(f(A))\right) \subseteq f^{-1}(\operatorname{cl}(f(A))) .
$$

But $w s C(A) \subseteq w s C\left(f^{-1}(f(A))\right)$. Therefore $f(w s C(A)) \subseteq c l(f(A))$.

6 . $\Rightarrow 7$. Let $B$ be a subset of $Y$. By 6 ,

$$
f\left(w s C\left(X \backslash f^{-1}(B)\right)\right) \subseteq \operatorname{cl}\left(f\left(X \backslash f^{-1}(B)\right)\right)
$$

and

$$
f\left(X \backslash w s I\left(f^{-1}(B)\right)\right) \subseteq \operatorname{cl}(Y \backslash B)=Y \backslash \operatorname{int}(B) .
$$

Therefore $X \backslash w s I\left(f^{-1}(B)\right) \subseteq f^{-1}(Y \backslash \operatorname{int}(B))$ and $f^{-1}(\operatorname{int}(B)) \subseteq w s I\left(f^{-1}(B)\right)$.

7. $\Rightarrow 1$. Let $B$ be an open in $Y$ and $f^{-1}(\operatorname{int}(B)) \subseteq w s I\left(f^{-1}(B)\right)$. Then

$$
f^{-1}(B) \subseteq w s I\left(f^{-1}(B)\right) .
$$

But $w s I\left(f^{-1}(B)\right) \subseteq f^{-1}(B)$. Hence $f^{-1}(B)=w s I\left(f^{-1}(B)\right)$. Therefore $f^{-1}(B)$ is $w$-semi open. 
As immediate consequence of Theorem 4.5, we have the following result.

Corollary 4.6. Let $\left(X, w_{X}, \tau\right)$ be a weak topological space and $(Y, \sigma)$ be a topological space and $f:(X, \tau) \rightarrow(Y, \sigma)$ a $(w, \sigma)$-s-continuous function, then the following are equivalent:

1. $w s I\left(w s C\left(f^{-1}(B)\right)\right) \cap w s C\left(w s I\left(f^{-1}(B)\right)\right) \subseteq f^{-1}(\operatorname{cl}(B))$ for each $B$ in $Y$.

2. $f[w s I(w s C(A)) \cap w s C(w s I(A))] \subseteq c l(f(A)))$ for each $A$ in $X$.

Definition 4.7. Let $\left(X, w_{X}, \tau\right)$ be a weak topological space and $A \subseteq X$. Then the $w$-s-kernel of $A$ denoted by $w-s-\operatorname{Ker}(A)$ is defined to be the set, $w-s-\operatorname{Ker}(A)=\cap\{U: U \in w S O(X), A \subseteq U\}$.

Theorem 4.8. Let $\left(X, w_{X}, \tau\right)$ be a weak topological space and $x \in X$. Then $y \in w-s-\operatorname{Ker}(\{x\})$ if and only if $x \in w s C(\{y\})$.

Proof. Assume that $y \notin w-s-K \operatorname{er}(\{x\})$. Then there exists a $w$-semi open set $U$ containing $x$ such that $y \notin U$. Therefore, we have $x \notin w s C(\{y\})$. The converse is similarly shown.

Theorem 4.9. Let $\left(X, w_{X}, \tau\right)$ be a weak topological space and $A$ a subset of $X$. Then $w-s-\operatorname{Ker}(A)=\{x \in X: w s C(\{x\}) \cap A \neq \emptyset\}$.

Proof. Let $x \in w-s-\operatorname{Ker}(A)$ and $w s C(\{x\}) \cap A=\emptyset$. Then, $x \notin X \backslash w s C(\{x\})$ which is a $w$-semi open set containing $A$. But this is impossible, since $x \in w$-s$\operatorname{Ker}(A)$. Consequently, wsC $(\{x\}) \cap A \neq \emptyset$.

Conversely, let $x \in X$ such that $w s C(\{x\}) \cap A \neq \emptyset$. Suppose that $x \notin w$-s$\operatorname{Ker}(A)$. Then there exists a $w$-semi open set $U$ containing $A$ and $x \notin U$. Let $y \in w s C(\{x\}) \cap A$. Then $y \in w s C(\{x\})$ and $y \in A$. Thus $x \in w-s-\operatorname{Ker}(\{y\})$ and $y \in U \in w$ implies $x \in U \in S O(X)$. By this contradiction, $x \in w-s-\operatorname{Ker}(A)$.

Theorem 4.10. The following are equivalent for any points $x$ and $y$ in a weak space $\left(X, w_{X}\right)$ :

1. $w-s-\operatorname{Ker}(\{x\}) \neq w-s-\operatorname{Ker}(\{y\})$.

2. $w s C(\{x\}) \neq w s C(\{y\})$.

Theorem 4.11. Let $\left(X, w_{X}, \tau\right)$ be a weak topological space and $A \subseteq X$. Then

1. $x \in w-s-\operatorname{Ker}(A)$ if and only if $A \cap F \neq \emptyset$ for any $w$-semi closed subset $F$ of $X$ with $x \in F$. 
2. $A \subseteq w-s-\operatorname{Ker}(A)$ and $A=w$-s-Ker $(A)$ if $A$ is $w$-semi open in $X$.

3. If $A \subseteq B$ then $w-s-\operatorname{Ker}(A) \subseteq w-s-\operatorname{Ker}(B)$.

Proof. 1. Let $x \in w-s-\operatorname{Ker}(A)$. Then by Theorem 4.9, $A \cap w s C(\{x\}) \neq$ $\emptyset$. Conversely, assume that $A \cap F \neq \emptyset$. By taking $F=w s C(\{x\})$, we have $A \cap w s C(\{x\}) \neq \emptyset$ which implies $x \in w$-s-Ker $(A)$.

2. Let $A$ be $w$-semi open in $X$. Then always $A \subseteq w$-s-Ker $(A)$. On the other hand, assume that $x \in w-s-\operatorname{Ker}(A)$. Then $x \in$ $\bigcap\{U: U \in w S O(X), A \subseteq U\}$. Since $A$ is $w$-semi open implies that $x \in A$. Thus $w$-s-Ker $(A) \subseteq A$. Hence $A=w-s-\operatorname{Ker}(A)$.

3. It is obvious.

As immediate consequence of Theorems 4.5 and 4.11 , we have the following result.

Corollary 4.12. Let $\left(X, w_{X}, \tau\right)$ be a weak topological space, $(Y, \sigma)$ be a topological space and $f:(X, \tau) \rightarrow(Y, \sigma)$ a function $(w, \sigma)$-s-continuous. Then the following are equivalent :

1. For every subset $A$ of $X, f(w s I(A)) \subset \operatorname{Ker}(f(A))$.

2. For every subset $B$ of $Y, w s I\left(f^{-1}(B)\right) \subseteq f^{-1}(\operatorname{Ker}(B))$.

Definition 4.13. Let $\left(X, w_{X}, \tau\right)$ be a weak topological space and $(Y, \sigma)$ be a topological space. Then $f:(X, \tau) \rightarrow(Y, \sigma)$ is said to be gw-s-continuous (respectively contra locally $w$-s-continuous) if $f^{-1}(F)$ is a gw-s-closed (respectively locally $w$-s-closed) for each closed set $F$ of $(Y, \sigma)$.

Example 4.14. In Example 3.13, take $f:(X, \tau) \rightarrow(X, \tau)$, defined as: $f(a)=b, f(b)=c$ and $f(c)=a$, then $f$ is contra locally $w$-s-continuous but is not gw-s-continuous. In the same form if in Example 3.25, we define $f:(X, \tau) \rightarrow(X, \tau)$, as: $f(a)=b, f(b)=a$ and $f(c)=c$, then $f$ is $w$-Scontinuous but is not contra locally $w$-s-continuous. Observe that in each case $\mathrm{f}$ is not $(w, \tau)$-s-continuous.

The following theorem is a direct consequence of Theorem 4.5 and Theorem 3.22

Theorem 4.15. Let $\left(X, w_{X}, \tau\right)$ be a weak topological space and $(Y, \sigma)$ be a topological space. Then $f:(X, \tau) \rightarrow(Y, \sigma)$ is $(w, \sigma)$-s-continuous if and only if it is gw-s-continuous and contra locally $w$-s-continuous. 
The following example shows the existence of a function that is contra locally $w$-s-continuous but not is gw-s-continuous, in consequence is not $(w, \tau)$ s-continuous.

Example 4.16. In Example 3.12, define $f:(X, \tau) \rightarrow(X, \tau)$ as follows: $f(a)=a, f(b)=d, f(c)=b$ and $f(d)=c$. According with 3.20, $f$ is contra locally $w$-s-continuous but not is gw-s-continuous, in consequence is not $(\mu, \tau)$ s-continuous.

Theorem 4.17. Let $\left(X, w_{X}, \tau\right)$ be a weak topological space and $(Y, \sigma)$ be a topological space. Then a contra continuous function $f:(X, \tau) \rightarrow(Y, \sigma)$ is $(w, \sigma)$-s-continuous if and only if it is gw-s-continuous

Proof. Suppose that $f$ is contra continuous and $(w, \sigma)$-s-continuous. Let $F$ be a closed set in $Y$, then $f^{-1}(F)$ is open and $w$-semi closed in $X$. Since each $w$-semi closed is gw-s-closed, then $f$ is g $w$-s-continuous.

Conversely, let $F$ be a closed set in $Y$, then $f^{-1}(F)$ is open and gw-s-closed in $X$. Since each open set is locally $w$-s-closed, then $f^{-1}(F)$ is locally $w$-s-closed and gw-s-closed, by Theorem 3.22, $f$ is $(w, \sigma)$-s-continuous.

Example 4.18. In Example 4.4, $f$ is $(\mu, \sigma)$-s-continuous, $f(\mathbb{R} \backslash \mathbb{Q})=\mathbb{R} \backslash \mathbb{Q}$ is $w$-open but is not $w$-s-closed in consequence, $f$ is not contra continuous.

\section{References}

[1] A. Császar, Generalized topology, generalized continuity, Acta Math. Hungar., 96 (2002), 351-357, doi: 10.1023/A:1019713018007.

[2] A. Császar, Generalized open sets in generalized topologies, Acta Math. Hungar., 96 (2005), 53-66, doi: 10.1007/s10474-005-0005-5.

[3] A. Császar, $\delta$ and $\theta$-modifications of generalized topologies, Acta Math. Hungar., 120, No. 3 (2008), 275-279, doi: 10.1007/s10474-007-7136-9.

[4] Bishwambhar Roy and Ritu Sen. On a type of decomposition of continuity, Afr. Mat., 26, No. 1-2 (2015), 153-158, doi: 10.1007/s13370-013-0195-x.

[5] Bishwambhar Roy and Ritu Sen. On decomposition of weak continuity, Creat. Math. Inform., 24, No. 1-2 (2015), 83-88.

[6] W. K. Min, On weakly $w_{\tau} g$-closed sets in associated $w$-spaces, International Journal of Pure an Applied Mathematics, 113, No. 1 (2017), 181-188, doi: 10.12732/ijpam.v112i2.18.

[7] W. K. Min and Y. K. Kim, On weak structures and $w$-spaces, Far East Journal of Mathematical Sciences, 97, No. 5 (2015), 549-561.

[8] W. K. Min and Y. K. Kim, w-semi open sets and $w$-semi continuity in weak spaces, International Journal of Pure an Applied Mathematics, 110, No. 1 (2016), 49-56, doi: 10.12732/ijpam.v110i1.5. 
[9] E. Rosas, C. Carpinteron, John Moreno and J. Sanabria, Decomposition of weak continuous Functions, (2017), Submitted.

[10] Carlos Carpintero, John Moreno and Ennis Rosas, Some New types of decomposition of continuity II, Creat. Math. Inform., 25 No. 1 (2016), 41-49. 